cellular matrix, resulting in loss of airway or alveolar function. Murine lineage tracing experiments have suggested that these fibroblasts may, in part, be derived from lung epithelium via epithelial to mesenchymal transition (EMT). Transforming Growth Factor- $\beta 1$ (TGF- $\beta 1$ ) has been shown to drive EMT both in vitro and in vivo. Previous work in our lab has shown that inflammatory stimuli such as Tumour Necrosis Factor- $\alpha(T N F \alpha)$ can accentuate TGF- $\beta 1$ driven EMT in primary bronchial epithelial cells (PBEC). This crosstalk between inflammatory and TGF- $\beta 1$ signalling may have implications in the pathogenesis of fibrotic disorders of the lung including Bronchiolitis Obliterans Syndrome and Idiopathic Pulmonary Fibrosis. We hypothesised that TGF- $\beta$ activated kinase-1 (TAK-1), a protein phosphorylated in response to both TGF- $\beta 1$ and TNF $\alpha$, may be a key convergent point and assessed its role in inflammatory accentuation of EMT.

Methods PBEC isolated from stable lung transplant recipients were treated with TGF- $\beta 1(10 \mathrm{ng} / \mathrm{ml})$ and/or TNF $\alpha(20 \mathrm{ng} / \mathrm{ml})$ and the phosphorylation and localisation of signalling molecules SMAD-3 and TAK-1 assessed (western blot, confocal microscopy). TAK-1 function was blocked using a selective inhibitor and by siRNA knockdown; effects on EMT marker expression were assessed (western blot, confocal microscopy).

Results TAK- 1 is phosphorylated in response to both TGF- $\beta 1$ (161\% increase) and TNFa (145\%) with an accentuated phosphorylation upon co-treatment $(516 \%, n=3)$. Co-treatment also resulted in pronounced nuclear localisation of phosphorylated TAK-1, which has not previously been reported. Chemical Inhibition of TAK-1 phosphorylation significantly reduced TGF- $\beta 1$ driven down-regulation of E-cadherin ( $22 \%$ inhibition) and up-regulation of Vimentin $(78 \%)$ and Fibronectin (39\%, $\mathrm{p}<0.05, \mathrm{n}=4)$. Furthermore, TAK-1 inhibition significantly reduced TNF $\alpha$ accentuated TGF- $\beta 1$ driven down-regulation of E-cadherin (39\%) and up-regulation of Fibronectin $(87 \%)$ and Vimentin $(258 \%, \mathrm{p}<0.05, \mathrm{n}=4)$. These findings were independently validated via siRNA knockdown of TAK-1.

Conclusions These findings suggest that TAK- 1 is an important convergent point for inflammatory and TGF- $\beta 1$ signalling, controlling the accentuation of EMT in primary human airway epithelium. Examination of TAK-1 activation and function in animal models of lung fibrosis may provide information on the potential for TAK-1 inhibition as a therapeutic target.

\section{S141 A DISINTEGRIN AND METALLOPROTEASE (ADAM) 33 PROTEIN IN PATIENTS WITH PULMONARY SARCOIDOSIS}

doi:10.1136/thx.2010.150946.42

${ }^{1} \mathrm{~A} S$ Shaffiq, ${ }^{1} \mathrm{H} \mathrm{M} H$ Haitchi, ${ }^{1} \mathrm{Y} Y \mathrm{P}$ Pang, ${ }^{2} \mathrm{~A}$ A Alangari, ${ }^{1} \mathrm{M} \mathrm{G} J$ Jones, ${ }^{1} \mathrm{~B}$ G M Marshall, ${ }^{1} \mathrm{~K}$ M A 0 O'Reilly, ${ }^{1} \mathrm{D}$ E D Davies. ${ }^{1}$ University of Southampton School of Medicine, Southampton, UK; ${ }^{2}$ King Saud University, Riyadh, Saudi Arabia

Background The asthma and chronic obstructive pulmonary disease (COPD) gene, A Disintegrin And Metalloproteinase (ADAM)33, is selectively expressed in mesenchymal cells and its metalloprotease activity has been linked to angiogenesis and airway remodelling. A soluble form of ADAM33 (sADAM33) has been identified in the bronchoalveolar lavage fluid (BALF) of asthmatic patients and its levels inversely correlate with lung function and disease severity. Because tissue remodelling also occurs in pulmonary sarcoid, we hypothesised that sADAM33 is elevated in BALF of patients with this disease which, like asthma, is heterogeneous.

Methods BALF was obtained from healthy controls $(n=11)$ and patients with sarcoid $(n=13)$ using fibre optic bronchoscopy according to current guidelines. After removal of immunoglobulins using Protein $\mathrm{A} / \mathrm{G}$ and enrichment using Concanavalin $\mathrm{A}$ beads, sADAM33 was identified in BALF by Western blotting. A FRET peptide cleavage assay was used to assess ADAM33-like activity in BALF. Lung function (FVC\%) and gas transfer (TLCO\%) were measured at time of first diagnostic workup.
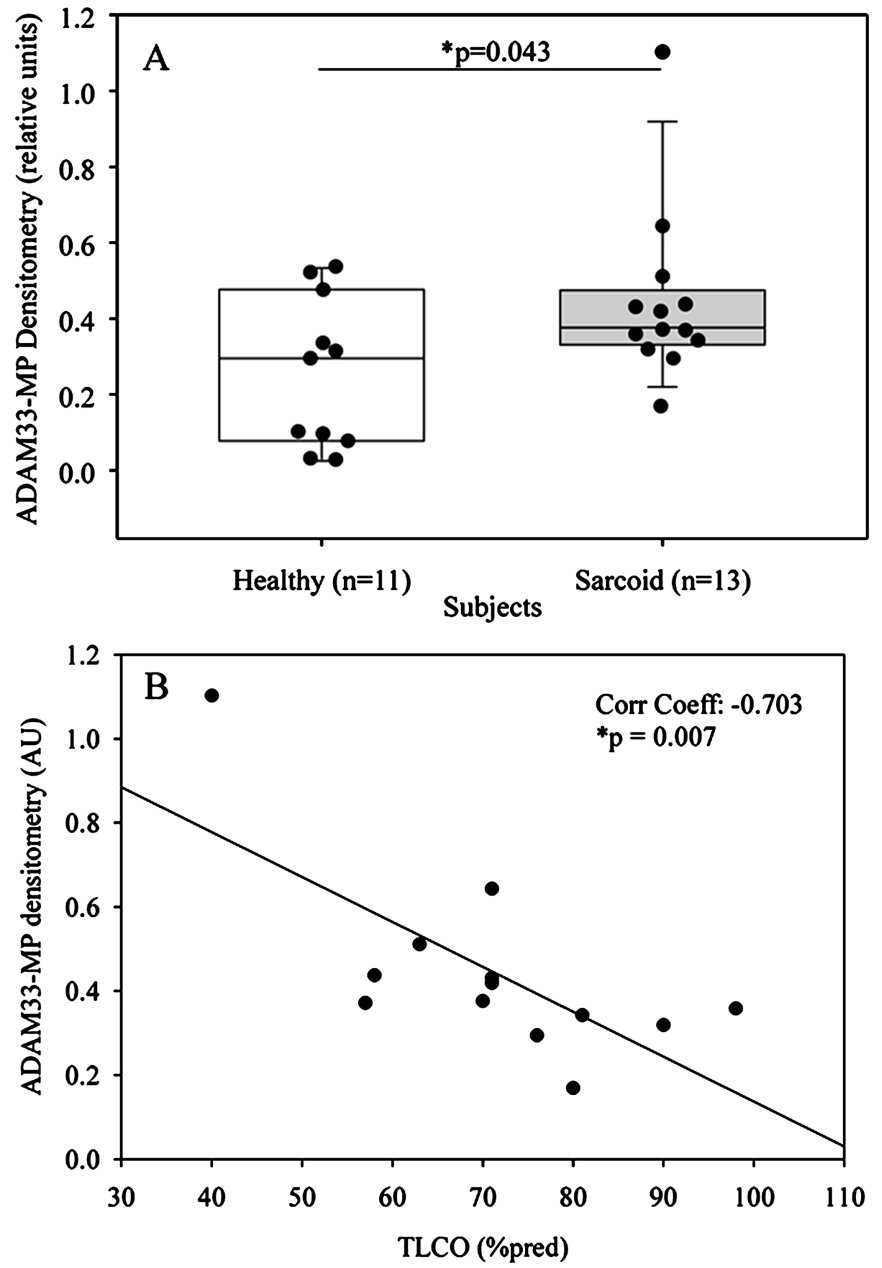

Abstract S141 Figure 1

Results sADAM33 protein in BALF was detected as a $25 \mathrm{kDa}$ fragment and levels were significantly increased in samples from sarcoid patients when compared to healthy controls $(p<0.05)$ (Abstract S141 Figure 1A). Levels of sADAM33 were inversely correlated with lung function (FVC $\%$ predicted) $(\mathrm{p}<0.05)$ and gas transfer $($ TLCO $\%$ predicted) $(p<0.01)$ (Abstract S141 Figure 1B). No difference in sADAM33 enzymatic activity was observed between healthy and sarcoid BALF samples.

Conclusion Release of sADAM33 is increased in sarcoid in association with abnormal lung function. Further studies will be required to determine whether the release of sADAM33 results in dysregulated metalloprotease activity, leading to angiogenesis and pulmonary parenchymal remodelling in pulmonary sarcoid. Since ADAM33 polymorphism is related to reduced lung function in asthma and COPD, this study raises the possibility that there may also be genetic associations between $A D A M 33$ and some forms of pulmonary sarcoid. Finally, the occurrence of sADAM 33 in asthma and sarcoidosis and its relation to reduced lung function suggests that it may be a biomarker of pulmonary remodelling in these diseases.

\section{S142 THE $\mathrm{K}^{+}$CHANNEL KCa3.1 IS EXPRESSED IN HUMAN LUNG FIBROBLASTS}

doi:10.1136/thx.2010.150946.43

${ }^{1} \mathrm{~K}$ Roach, ${ }^{1} \mathrm{G}$ Arthur, ${ }^{2} \mathrm{C}$ Feghali-Bostwick, ${ }^{3} \mathrm{~W}$ Coward, ${ }^{1} \mathrm{~S} \mathrm{M}$ Duffy, ${ }^{1} \mathrm{P}$ Bradding ${ }^{1}$ Institute for Lung Health, Department of Infection, Immunity and Inflammation, University of Leicester, Leicester, UK; ${ }^{2}$ Department of Medicine, Division of Pulmonary, Allergy, and Critical Care Medicine, University of Pittsburgh, Pittsburgh, USA; ${ }^{3}$ Division 